\title{
Uso do cateter vesical de demora em uma unidade de terapia intensiva: estudo transversal
}

\author{
Use of indwelling bladder catheters in an intensive care unit: cross-sectional study
}

El uso del catéter vesical de larga duración en una unidad de cuidados intensivos: estudio transversal

\author{
Caroline da Costa Silva de Azevedo' @; Luana Ferreira de Almeidal @; Camila Tenuto Messias da Fonseca ${ }^{\prime}$;

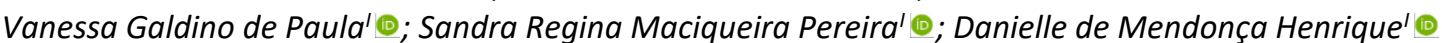

'Universidade do Estado do Rio de Janeiro, Rio de Janeiro, RJ, Brasil

\begin{abstract}
RESUMO
Objetivo: analisar o uso de cateter vesical de demora em unidade de terapia intensiva. Método: estudo observacional transversal, descritivo, documental, realizado em uma unidade de terapia intensiva no Rio de Janeiro, entre maio e agosto de 2020. Amostra por conveniência, composta por 190 prontuários de pacientes com esse dispositivo. Utilizou-se questionário relacionado à caracterização dos pacientes, critérios de inserção; boas práticas; complicações não infecciosas. Os dados foram analisados pela estatística descritiva, apresentados em frequência absoluta e relativa. Para eventos não infecciosos, foi realizado o Teste Exato de Fischer, com auxílio do programa Statistical Package for Social Sciences. Resultados: a inserção de cateter vesical de demora se deu majoritariamente no sexo feminino (123-64,7\%), pacientes com média de 62,9 anos e diagnóstico de COVID 19 (97 - 51,1\%). Em 134 (70,5\%) dos casos, os critérios foram atendidos. Conclusão: reforça-se a importância do cumprimento de protocolos e boas práticas no uso do cateter vesical de demora.
\end{abstract}

Descritores: Unidades de Terapia intensiva; Cuidados de Enfermagem; Cateterismo Urinário; Segurança do Paciente.

\begin{abstract}
Objective: to analyze the use of indwelling bladder catheters in an intensive care unit. Method: this descriptive, cross-sectional, documentary study was conducted in an intensive care unit in Rio de Janeiro, between May and August 2020 with a convenience sample, comprising 190 medical records of patients with such devices. A questionnaire was used to characterize the patients, insertion criteria, best practices and non-infectious complications. The data were analyzed by descriptive statistics, presented in absolute and relative frequencies. For non-infectious events, Fischer's Exact Test was performed using the Statistical Package for Social Sciences. Results: indwelling bladder catheters were inserted mostly in females (123; 64.7\%), patients with mean age 62.9 years, and diagnosis of COVID 19 (97; 51.1\%). In 134 (70.5\%) of cases, the criteria were met. Conclusion: the findings underline the importance of compliance with protocols and good practices when using indwelling bladder catheters.

Descriptors: Urinary Catheterization; intensive care units; Nursing Care; Urinary Catheterization; Patient Safety.
\end{abstract}

\section{RESUMEN}

Objetivo: analizar el uso del catéter vesical de larga duración en una unidad de cuidados intensivos. Método: estudio observacional, transversal, descriptivo, documental, realizado en una unidad de cuidados intensivos en Río de Janeiro, entre mayo y agosto de 2020. Muestra de conveniencia, compuesta por 190 historias clínicas de pacientes con este dispositivo. Se utilizó un cuestionario relacionado con la caracterización de los pacientes, criterios de inserción; buenas prácticas; complicaciones no infecciosas. Los datos fueron analizados por estadística descriptiva, presentados en frecuencia absoluta y relativa. Para los eventos no infecciosos, se realizó la Prueba Exacta de Fischer, con la ayuda del programa Statistical Package for Social Sciences. Resultados: la inserción de catéter vesical de larga duración se produjo principalmente en mujeres (12364,7\%), pacientes con media de edad de 62,9 años y diagnóstico de COVID 19 (97 - 51,1\%). En 134 (70,5\%) de los casos, se cumplieron los criterios. Conclusión: Se refuerza la importancia del cumplimiento de protocolos y buenas prácticas en el uso del catéter vesical de larga duración.

Descriptores: Unidades de Cuidados Intensivos; Atención de Enfermería; Cateterismo Urinario; Seguridad del Paciente.

\section{INTRODUÇÃO}

Na unidade de terapia intensiva (UTI), em especial pela instabilidade hemodinâmica dos pacientes, uso de drogas vasoativas e necessidade rigorosa do controle da diurese, o cateterismo urinário corresponde a um procedimento usual ${ }^{1}$.

Um estudo com 433 pacientes internados em uma UTI, demonstrou que 93,3\% utilizaram o cateter vesical de demora (CVD) por mais de 24 horas $^{2}$. Outro estudo, também em UTI, evidenciou uma incidência da sua inserção em $86,74 \%$ dos pacientes ${ }^{3}$.

Embora indicado em casos específicos, a inserção e manutenção desse dispositivo médico apresenta riscos e complicações infecciosas e não infecciosas, como a infecção do trato urinário associada ao cateter vesical de demora (ITU-AC) e o trauma uretral ${ }^{4}$.

Autora correspondente: Luana Ferreira de Almeida. E-mail: luana.almeida3011@gmail.com

Editora Científica: Cristiane Helena Gallasch; Editora Associada: Flavia Giron Camerini 
Dados do Boletim de Segurança do Paciente e Qualidade em Serviços de Saúde no22 publicado em 2020, que analisou os Indicadores Nacionais de Infecção Relacionada à Assistência à Saúde (IRAS) e Resistência Antimicrobiana (RM) referente ao ano de 2019, apontam que no Brasil, ocorrem 3,6 casos de ITU-AC por 1000 dias de uso de cateter vesical ${ }^{5}$.

Já a presença do trauma uretral pode manifestar-se devido à inserção do cateter de maneira indevida ou pelo atrito do cateter mal lubrificado, e ocasionar outras complicações como uretrorragias e ITU-AC ${ }^{6}$.

Assim, tendo em vista à prevenção das complicações relacionadas ao uso de CVD, no Brasil preconiza-se o seguimento de critérios para sua a utilização. São eles: impossibilidade de micção espontânea, instabilidade hemodinâmica com necessidade de monitorização do débito urinário, pós-operatório de cirurgias não urológicas em até 24 horas, cirurgias urológicas específicas, presença de lesão por pressão (LP) estágio 4 em pacientes do sexo feminino com incontinência urinária ${ }^{7}$.

Nessa direção, torna-se relevante investigar o uso do dispositivo, possibilitando intervenções em pontos que possam se apresentar mais frágeis, e assim propor estratégias para o uso seguro desse dispositivo e redução nas taxas de complicações a ele relacionadas.

Diante do exposto, o presente estudo teve como objetivo: Analisar o uso de cateter vesical de demora em pacientes críticos em unidade de terapia intensiva.

\section{REVISÃO DE LITERATURA}

Os cuidados de enfermagem com o uso do CVD envolvem recomendações bem definidas quanto à sua indicação, boas práticas durante a instalação e manutenção do dispositivo, e retirada precoce ${ }^{7-9}$.

Durante o procedimento da sua inserção podem ocorrer complicações não infecciosas, como o trauma uretral, a dor e o falso trajeto ${ }^{6}$. Ocasionalmente, podem ser acompanhadas de uretrorragias significativas. Além disso, após 72 horas de cateterização aumentam os riscos de ITU-AC ${ }^{10}$.

Aproximadamente $17 \%$ a $69 \%$ de ITU-AC pode ser evitada a partir de recomendações de boas práticas que busquem diminuir a taxa de complicações pelo uso do $C V D^{8}$, como higienização das mãos, rigor na técnica de inserção, manutenção e a forma como o cateter é removido ${ }^{11}$.

De acordo com o Centers for Disease Control and Prevention (CDC), a sua utilização pode chegar em $15 \%$ a $25 \%$ em pacientes internados, sendo indicado de acordo com as condições clínicas do paciente, com tempo de permanência avaliado diariamente, conforme sua necessidade ${ }^{8,12}$.

Vale ressaltar que as recomendações do Institute for Healthcare Improvement (IHI), para cateterismo vesical relacionam-se à necessidade de monitoramento do débito urinário em pacientes críticos, pacientes com retenção ou obstrução urinária aguda, procedimentos cirúrgicos específicos, no auxílio da cicatrização de LP em pacientes incontinentes ou nos cuidados de fim de vida para o conforto do paciente ${ }^{13}$, conforme às recomendações da ANVISA ${ }^{7}$. A introdução do cateter deve ser realizada pelo enfermeiro, através de técnica asséptica, sendo importante a orientação da equipe quanto à manipulação e cuidados com esse dispositivo ${ }^{14}$.

É indicada higienização das mãos em todos os momentos de manipulação do sistema ou do sítio de inserção; higiene rotineira do meato uretral; manutenção da bolsa coletora abaixo do nível da bexiga; esvaziamento da bolsa coletora regularmente; utilização do coletor individual, evitando o contato do tubo de drenagem com o recipiente coletor; e troca de todo sistema quando ocorrer desconexão, quebra da técnica asséptica ou vazamento ${ }^{15}$.

A fixação deste dispositivo evita o trauma uretral; sendo indicado, nas mulheres, a fixação na face inferior da coxa; e nos homens, na parte inferior do abdome ${ }^{16}$. Outra medida relevante corresponde à identificação da bolsa coletora de diurese, pois contribui para o controle do uso do dispositivo ${ }^{17}$.

Para além das ações citadas, recomenda-se a implementação de processos de qualidade e segurança como garantia de boas práticas, tais como uso de bundle de inserção e manutenção, incluindo a padronização do protocolo de cuidados relacionados ao uso de CVD e seus indicadores ${ }^{18}$.

Também merece destaque a conscientização da equipe de enfermagem, como realização de educação continuada a esses profissionais sobre os cuidados com o CVD, para a prevenção de IRAS e outras complicações ${ }^{18}$.

\section{MÉTODO}

Estudo observacional, com desenho transversal, descritivo, documental, seguindo os vinte e dois itens do método $\mathrm{STROBE}^{19}$, realizado em uma UTI geral de adulto em um hospital universitário, no município do Rio de Janeiro, no período de maio a agosto de 2020 . 
A amostra foi por conveniência, composta por 190 pacientes internados na unidade investigada, durante a realização do estudo.

Foram incluídos no estudo todos os pacientes em uso de CVD, instalados antes da admissão ou durante a permanência na unidade investigada, com tempo de internação na UTI maior que 24 horas. Sendo assim, não foram considerados critérios de exclusão entre os pacientes em uso de CVD.

Os dados foram coletados a partir de informações registradas no prontuário do paciente, através de um formulário elaborado conforme recomendações da ANVISA ${ }^{7}$ quanto ao uso do CVD. As variáveis investigadas se relacionaram com: a) as características dos pacientes, incluindo diagnóstico, sexo, idade e uso de medicamentos (anticoagulantes, antimicrobianos, diuréticos e aminas vasoativas); b) critérios de inserção de CVD como a impossibilidade de micção espontânea, instabilidade hemodinâmica com necessidade de monitorização de débito urinário, pós-operatório até 24h, cirurgia urológica, sexo feminino em tratamento de lesão por pressão estágio 4 com cicatrização comprometida pela urina; c) boas práticas relacionadas à inserção e manutenção do cateter, como a fixação do cateter, registro da data de inserção e da retirada do CVD; e d) eventos não infecciosos relacionadas ao uso de CVD, sendo investigados presença coágulos no circuito da bolsa coletora, presença de grumos e lesão relacionada ao dispositivo.

Os dados relativos às características dos pacientes e critérios de inserção de CVD, foram analisados pela estatística descritiva, sendo expressos em frequência absoluta e relativa. Para análise dos eventos não infecciosos, foi realizado o Teste Exato de Fischer, calculado com auxílio do programa Statistical Package for Social Sciences (SPSS) versão 20,0, para associação do uso de anticoagulantes e fixação do cateter, com as complicações não infecciosas apresentadas pelos pacientes.

O estudo foi aprovado pelo Comitê de Ética e Pesquisa da instituição no dia 30 de março de 2020, sob parecer número 4.283.055.

\section{RESULTADOS E DISCUSSÃO}

Foram analisados 190 prontuários, o diagnóstico de COVID-19 foi confirmado em 97 (51,1\%) dos casos. Considerando o período de pandemia do vírus SARS CoV-2, a UTI onde foi realizada a pesquisa tornou-se unidade coorte para pacientes com suspeita ou diagnóstico da doença, correspondendo a um ambiente para tratamento de pacientes críticos, pois dispõe de recursos tecnológicos, suporte ventilatório e de profissionais capacitados. Dados de um estudo apontaram que a oxigenoterapia foi necessária em 44,4\% dos pacientes hospitalizados com COVID -19, e 23,6\% deles demandaram ventilação mecânica, necessitando de internação em UTI20.

Entre os pacientes que utilizaram CVD, predominou o sexo feminino ( $n=123 ; 64,7 \%$ ), a média de idade foi $62,9( \pm 14,83)$ anos, variando entre 29 e 95 anos, corroborando com outras pesquisas nas quais as internações na UTI são prevalentes na população com idade maior que 60 anos ${ }^{21,22}$. Esse dado relaciona-se também com o processo de envelhecimento no Brasil e com estudos sobre a epidemiologia da COVID-19, que demonstraram prevalência de 60 anos entre os pacientes acometidos pelo coronavírus ${ }^{23,24}$.

Quanto a indicação correta do uso de CVD, em relação aos critérios de inserção recomendados na literatura7, estes, foram atendidos em 134 (70,5\%) das situações investigadas. Em 92 (48,4\%), os pacientes atendiam a três critérios, em $27(14,2 \%)$ a dois, e em 15 (7,9\%) a um, indo de encontro com estudo que refere que a utilização de CVD, em algum momento da internação na UTI, é realizado por aproximadamente $12 \%$ a $16 \%$ dos pacientes internados nesse setor ${ }^{25}$.

Na Tabela 1 é apresentada a distribuição dos critérios de inserção do CVD encontrada nesse estudo.

TABELA 1: Distribuição dos critérios de inserção do CVD, conforme recomendação da Anvisa ( $n=190)$. Rio de Janeiro, $\mathrm{RJ}$, Brasil, 2020.

\begin{tabular}{lcc}
\hline Variáveis & $\mathbf{n}$ & $\mathbf{\%}$ \\
\hline Impossibilidade de micção espontânea & 116 & 61,1 \\
Sim & 74 & 38,9 \\
Não & & \\
Instabilidade hemodinâmica com necessidade & 1 & 0,5 \\
Sim & 189 & 99,5 \\
Não & & \\
Cirurgia urológica & - & - \\
Sim & 190 & 100 \\
Não & & \\
Paciente de sexo feminino com LP grau IV & - & - \\
Sim & 190 & 100 \\
Não
\end{tabular}


Quanto à impossibilidade de micção espontânea, uma pesquisa mostrou que 6,4\% dos pacientes internados possuíam esse critério para inserção do $\mathrm{CVD}^{26}$, contrapondo os resultados desse estudo ( $\mathrm{n}=116 ; 61,1 \%$ ). Pode-se compreender esse resultado, pois a UTI é destinada a pacientes em estado grave, que necessitam de sedação e analgesia para promover conforto durante a terapêutica ${ }^{27,28}$.

A instabilidade hemodinâmica foi critério para o uso de CVD ( $n=116 ; 61,1 \%)$. Em uma pesquisa com pacientes acometidos pelo novo coronavírus, $26 \%$ necessitaram de internação em uma UTI, devido ao agravamento da doença ${ }^{29}$. Tendo em vista a unidade investigada ser coorte durante a coleta de dados, pode-se entender que os critérios para uso de CVD, relacionados a pós-operatório até 24horas e pós-operatório de cirurgia urológica, não foram expressivos.

Tratando-se da indicação do uso de CVD em pacientes do sexo feminino com LP estágio 4, embora pesquisas mostrem que, na UTI, os pacientes possuíam alto risco para desenvolvimento de $\mathrm{LP}^{30}$, os dados mostraram que tal critério não apareceu, assim como em outros estudos ${ }^{31,32}$

Considerando a frequência com que a cateterização vesical é realizada nesse ambiente e o potencial de prevenção da ITU-AC, faz-se necessário a discussão entre equipe multiprofissional quanto aos riscos e benefícios do procedimento de cateterização vesical, sendo importante a avaliação diária da necessidade de permanência. Nas situações de incontinência e estabilidade hemodinâmica, a retenção urinária pode ser manuseada de outras formas. A realização do procedimento para evitar constantes trocas de roupas de camas, fraldas e para aliviar o trabalho da equipe e cuidados junto ao leito, não justifica a permanência do CVD.

Os enfermeiros devem incluir em suas visitas diárias, a conferência sistemática dos critérios a que estão submetidos os pacientes sob sua responsabilidade, discutir e registrar a justificativa para a manutenção do cateter vesical, buscando alternativas viáveis para conforto e com menor risco de infecção.

Além disso, cuidados como a escolha do cateter ideal, a habilidade na inserção, a garantia de uma fixação correta, evitando peso excessivo na bolsa de drenagem e prevenindo a retirada ou tração acidental, também são importantes na prevenção de complicações relacionadas ao cateterismo vesical de demora ${ }^{18}$.

Embora recomendada a identificação correta da bolsa coletora ${ }^{17}$, verificou-se $12(6,3 \%)$ registros das datas de inserção do CVD, contrapondo um estudo que demonstrou 96,2\% de conformidade dessa prática ${ }^{33}$.

Outra boa prática corresponde à fixação do dispositivo, que apesar de ser uma conduta simples, sua aplicabilidade é pouco realizada na prática assistencial diária, devendo ser realizada para evitar o tracionamento do dispositivo ${ }^{34,35}$. Dez $(5,3 \%)$ dispositivos estavam fixados, corroborando com resultados de pesquisa que mostrou menor conformidade relacionada à fixação ${ }^{34}$. Essa medida contribui para a prevenção do trauma da uretra, refluxo do conteúdo e risco de ITU-AC.

Quanto às complicações, observou-se a presença de grumos no cateter ( $n=44 ; 23,2 \%)$, lesão uretral $(14-7,4 \%)$, presença de coágulos no circuito $(8-4,2 \%)$ e lesão por dispositivo $(1-1,1 \%)$ (Tabela 2$)$.

TABELA 2: Eventos não infecciosos relacionados ao uso do CVD ( $n=190)$. Rio de Janeiro, RJ, Brasil, 2020.

\begin{tabular}{lcc}
\hline Eventos não infecciosos & $\mathbf{n}$ & $\mathbf{\%}$ \\
\hline Presença de grumos no circuito do cateter & & \\
Sim & 44 & 23,2 \\
Não & 146 & 76,8 \\
Lesão uretral & 14 & 7,4 \\
Sim & 176 & 92,6 \\
Não & & \\
Presença de coágulos no circuito do cateter & 8 & 4,2 \\
Sim & 182 & 95,8 \\
Não & & \\
Lesão por fixação do dispositivo & 2 & 1,1 \\
Sim & 188 & 98,9 \\
Não & &
\end{tabular}

Embora tenham sido encontrados presença de grumos ou coágulos no circuito do CVD, não houve comprometimento do fluxo urinário. É importante destacar que a obstrução do cateter pode levar à reserva urinária, criando um ambiente propício para proliferação de microrganismos ${ }^{36}$. 
Quanto às lesões de uretra, todas ocorreram em pacientes do sexo masculino, demonstrando o quanto é necessário o seguimento de bundles e a discussão de indicadores relacionados ao uso de CVD, tais como a realização de boas práticas na manutenção do dispositivo.

Na Tabela 3, observam-se dados relacionados ao uso de medicamentos pelos pacientes em uso de CVD.

\begin{tabular}{lcc} 
TABELA 3: Distribuição da & $\begin{array}{r}\text { amostra } \\
\text { segundo ao uso de medicamentos }\end{array}$ \\
(n=190). Rio de Janeiro, RJ, Brasil, 2020. \\
\hline Medicamentos & n & $\%$ \\
\hline Uso de anticoagulantes & & \\
Sim & 154 & 81,1 \\
Não & 36 & 18,9 \\
Uso de antimicrobianos & & \\
Sim & 142 & 74,7 \\
Não & 48 & 25,3 \\
Uso de aminas vasoativas & & \\
Sim & 111 & 58,4 \\
Não & 79 & 41,6 \\
Uso de diuréticos & & \\
Sim & 34 & 17,8 \\
Não & 156 & 82,2 \\
\hline
\end{tabular}

Verificou-se o uso de anticoagulantes ( $n=154 ; 81,1 \%)$, antimicrobianos ( $n=142 ; 74,7 \%)$, aminas vasoativas ( $n=111 ;$ $58,4 \%)$ e diuréticos $(n=34 ; 7,8 \%)$ pelos pacientes.

Quanto ao uso de anticoagulantes, os dados justificam-se, pois são utilizados para prevenção da trombose profunda, complicação comum em pacientes que permanecem por muito tempo restritos ao leito, como é o caso de pacientes graves ${ }^{37}$.

Em relação aos antimicrobianos, compreende-se o uso desses medicamentos, devido à sua gravidade clínica, procedimentos invasivos recebidos e exposição frequente aos microrganismos ${ }^{38}$. No entanto, não se pode perder de vista o uso irracional deste tipo de medicamentos, aumentando a vulnerabilidade do paciente às IRAS.

O uso de aminas vasoativas ( $n=111 ; 58,4 \%$ ), nos pacientes em UTI, pode ser compreendido pela presença de complicações relacionadas à instabilidade hemodinâmica. No entanto, seu uso pode acarretar a diminuição do fluxo sanguíneo renal, assim alterando o volume urinário. Nessa direção, a utilização de CVD se justifica pela necessidade do controle rigoroso da diurese ${ }^{1}$.

Quanto ao diurético, sua utilização se dá, sobretudo, quando diante da administração de terapia intravenosa; há risco de sobrecarga hídrica, dificultando o tratamento. Os resultados mostraram que foram pouco empregados nos pacientes que estavam com CVD, contrapondo os dados de uma pesquisa, no qual o diurético foi utilizado por $49 \%$ dos pacientes $^{39}$.

Ao investigar a associação entre a presença de coágulos com o uso de anticoagulante não foi identificado significância estatística $(p=0,648)$, o que ocorreu também ao associar a fixação do cateter com a presença de lesão por dispositivo $(p=0,103)$ (Tabelas 4 e 5$)$.

TABELA 4: Associação do uso de anticoagulante e presença de coágulo no circuito da bolsa coletora de diurese $(n=190)$. Rio de Janeiro, RJ, Brasil, 2020

\begin{tabular}{|c|c|c|c|c|c|c|c|}
\hline \multirow{3}{*}{ Uso de anticoagulante } & \multicolumn{7}{|c|}{ Presença de coágulo no circuido da bolsa coletora de diures } \\
\hline & \multicolumn{2}{|c|}{ Sim } & \multicolumn{2}{|c|}{ Não } & \multicolumn{2}{|c|}{ Total } & \multirow{2}{*}{$\mathbf{p}^{*}$} \\
\hline & $\mathbf{n}$ & $\%$ & $\mathbf{n}$ & $\%$ & $\mathbf{n}$ & $\%$ & \\
\hline Sim & 6 & 75,0 & 148 & 81,3 & 154 & 81,1 & \\
\hline Não & 2 & 25,0 & 34 & 18,7 & 36 & 18,9 & 0,648 \\
\hline Total & 8 & 100,0 & 182 & 100,0 & 190 & 100,0 & \\
\hline
\end{tabular}

* Teste Exato de Fisher considerando nível de significância de 0,05 
TABELA 5: Associação da fixação do CVD com a presença de lesão por dispositivo ( $n=190)$. Rio de Janeiro, RJ, Brasil, 2020.

\begin{tabular}{lccccccc}
\hline & \multicolumn{6}{c}{ Presença de lesão pelo dispositivo } \\
Fixação do CVD & \multicolumn{2}{c}{ Sim } & \multicolumn{2}{c}{ Não } & \multicolumn{2}{c}{ Total } & \multirow{2}{*}{ * } \\
& $\mathbf{n}$ & $\%$ & $\mathbf{n}$ & $\%$ & $\mathbf{n}$ & $\%$ & p \\
\hline Sim & 1 & 50,0 & 9 & 4,8 & 10 & 5,3 & \\
Não & 1 & 50,0 & 179 & 95,2 & 180 & 94,7 & 0,103 \\
Total & 2 & 100,0 & 188 & 100,0 & 190 & 100,0 & \\
\hline
\end{tabular}

Considerando o pequeno número da amostra entre os expostos, optou-se por utilizar o teste exato de Fisher para análise da associação entre estas variáveis, não identificando associação entre elas, conforme descrito nas tabelas 4 e 5. No entanto, recomenda-se a utilização das boas práticas para inserção e manutenção do dispositivo médico.

\section{Limitações do estudo}

Como limitações, destaca-se a fragilidade de consistência e confiabilidade das informações contidas nos prontuários, especialmente diante do contexto da pandemia por COVID-19, assim como ausência de registros de infecção de trato urinário relacionada a cateter, escassez de informações acerca do tempo de permanência do dispositivo, em razão de falhas de registros na inserção e retirada do dispositivo. Ademais, a escassez de estudos sobre o tema dificultou a comparação dos achados com os resultados de outras pesquisas, considerando que a literatura sobre CVD está orientada predominante para infecção.

\section{CONCLUSÃO}

Os dados mostraram que a inserção de CVD se deu majoritariamente em pacientes do sexo feminino, com média de 62,9 anos, com o diagnóstico de COVID 19. Observou-se a necessidade de melhoria na adesão aos critérios de inserção do dispositivo, como o reforço das boas práticas, com a implementação e seguimento de protocolos institucionais, capacitação de equipe e acompanhamento de indicadores, tais como taxa de inserção, conforme critérios preconizados, tempo de permanência, presença de fixação do dispositivo, presença de lesões e outras complicações.

Recomenda-se a investigação do uso deste tipo de dispositivo médico em diferentes contextos de saúde, a fim de contribuir para a análise de práticas profissionais relacionadas tanto à inserção, quanto à manutenção desse dispositivo médico.

\section{REFERÊNCIAS}

1. Melo EM, Oliveira TMM, Marques AM, Ferreira AMM, Silveira FMM, Lima VF. Patients' characterization in use of vasoactive drugs hospitalized in intensive care unit. Rev. Fund. Care Online [Internet]. 2016 [cited 2019 Sep 15]; 8(3):4898-904. DOI: http://dx.doi.org/10.9789/2175-5361.2016.v8i3.4898-4904.

2. Mota EC, Oliveira AC. Catheter-associated urinary tract infection: why do not we control this adverse event? Rev. esc. enferm. USP [Internet]. 2019 [cited 2020 Nov 03]; 53:e03452. DOI: https://doi.org/10.1590/s1980-220x2018007503452.

3. Rodrigues CN e Pereira DCA. Infections to assistance to health occurred at an intensive care unit. Rev. Investig. Bioméd. [Internet]. 2016 [cited 2019 Sep 02]; 8:41-51. Available from: http://www.ceuma.br/portalderevistas/index.php/RIB/article/view/28/27.

4. Mazzo A, Bardivia CB, Jorge BM, Souza Junior VD, Fumincellí L, Mendes IAC. Urinary catheterization delay: clinical practice. Enferm. Glob. [Internet]. 2015 [cited 2019 Jul 20]; 14(38):50-9. Available from: http://scielo.isciii.es/scielo.php?script=sci_arttext\&pid=S1695-61412015000200003\&lng=pt.

5. Agência Nacional de Vigilância Sanitária - ANVISA (BR). Boletim Segurança do Paciente e Qualidade em Serviços de Saúde no 22. Avaliação dos Indicadores Nacionais de Infecção Relacionada à Assistência à Saúde (IRAS) e Resistência Antimicrobiana (RM). Brasília: Agência Nacional de Vigilância Sanitária; 2019; [cited 2021 Jul 29] Available from: https://app.powerbi.com/view?r=eyJrljoiZjQ5ZDhjZmEtNDdhOC00MDk3LWFiNDEtNzg0MmE4MmE2MjlhliwidCI6ImI2N2FmMj NmLWMzZjMtNGQzNS04MGM3LWI3MDg1ZjVIZGQ4MSJ9\&pageName=ReportSectionac5c0437dbe709793b4b.

6. Lenz LL. Cateterismo vesical: cuidados, complicações e medidas preventivas. Arq. Catarin. Med. [Internet]. 2006 [cited 2021 Jan. 10]; 35(1):82-91. Available from: http://www.acm.org.br/revista/pdf/artigos/361.pdf.

7. Brasil. Agência Nacional de Vigilância Sanitária - ANVISA (BR). Medidas de prevenção de infecção relacionada à assistência à saúde, 2. ed., 2017, Brasília. Caderno 4. [cited 2019 Sep 27] Available from: http://antigo.anvisa.gov.br/documents/33852/3507912/Caderno+4++Medidas+de+Preven\%C3\%A7\%C3\%A3o+de+Infec\%C3\%A7\%C3\%A3o+Relacionada+\%C3\%A0+Assist\%C3\%AAncia+\%C3\%A0+Sa\% C3\%BAde/a3f23dfb-2c54-4e64-881c-fccf9220c373. 
8. Gould CV, Umscheid CA, Agarwal RK, Kuntz G, Pegues DA; Healthcare Infection Control Practices Advisory Committee. Guideline for prevention of catheter-associated urinary tract infections, 2009. Infect. Control Hosp. Epidemiol. [Internet]. 2010 [cited 2021 Jan 05]; 31(4):319-26. DOI: https://doi.org/10.1086/651091.

9. Saint S, Fowler KE, Sermak K, Gaies E, Harrod M, Holland P, et al. Introducing the no preventable harms campaign: creating the safest health care system in the world, starting with catheter-associated urinary tract infection prevention. Am J Infect Control. [Internet]. 2015 [cited 2021 Jan 16]; 43(3):254-9. DOI: https://doi.org/10.1016/j.ajic.2014.11.016.

10. Jorge BM, Mazzo A, Mendes IAC, Trevizan MA, Martins JCA. Urinary tract infection related to catheter use: integrative review. Rev. Enferm. Ref. [Internet]. 2013 [cited 2021 Jan 10]; serllI (3):15-23. Available from:

https://www.researchgate.net/publication/260838620_Urinary_tract_infection_related_to_catheter_use_integrative_review.

11. Andrade VLF, Fernandes FAV. Prevention of catheter-associated urinary tract infection: implementation strategies of international guidelines. Rev. Latino-Am. Enferm. [Internet]. 2016 [cited 2019 Ago 22]; 24:e2678. DOI: https://doi.org/10.1590/1518-8345.0963.2678.

12. Almeida MM, Lindoso AM, Pessoa RMC. Evidence in practice urinary catheterization. Reon Facema. [Internet]. 2016 [cited 2019 Oct. 04]; 2(2):196-201 Available from: http://www.facema.edu.br/ojs/index.php/ReOnFacema/article/view/87/48.

13. How-to Guide: Prevent Catheter-Associated Urinary Tract Infections. Cambridge, MA: Institute for Healthcare Improvement. [Internet]. 2011; [cited 2020 Nov 04]. Available from: http://www.ihi.org.

14. Flores VGT, Júnior MAF. Fatores de risco para infecção do trato urinário dos pacientes submetidos ao procedimento de cateterismo vesical de demora e suas implicações para a enfermagem. Rev. Cient. Link. Júnior [Internet]. 2012. [cited 2021 Jan 16]; 2(2) Available from: http://linkania.org/junior/article/view/48.

15. Ferreira LL, Azevedo LMN, Salvador PTCO, Morais SHM, Paiva RM, Santos VEP. Nursing Care in Healthcare-Associated Infections: A Scoping Review: Scoping review. Rev. Bras. Enferm. [Internet]. 2019 [cited 2020 Nov 02]; 72(2):476-83 DOI: https://doi.org/10.1590/0034-7167-2018-0418.

16. Potter PA, Perry AG, Hall A, Stockert PA. Fundamentos de Enfermagem. 8 ed. Rio de Janeiro: Guanabara Koogan, 2013.

17. Schran LS, Girardi C, Feldhaus C, Luz MAP, Bordin V, Oliveira JLC, Nicola AL. Conformidade na identificação de dispositivos utilizados na assistência hospitalar. Varia Scientia - Ciências da Saúde [Internet]. 2017 [cited 2020 Oct. 23]; 3(2):201-12; Available from: http://e-revista.unioeste.br/index.php/variasaude/article/view/17967.

18. Doreste FCPL, Souza ALL, Queiroz NR, Luna AA, Silva NCM, Souza PA. Patient Safety and Urinary Tract Infection Prevention Measures Related to Delay Vesical Catheterization. Rev. Enferm. Atual In Derme [Internet]. 2019 [cited 2021 Jan 10]; 89(27):18. Available from: https://www.revistaenfermagematual.com/index.php/revista/article/view/61.

19. Malta M, Cardoso LO, Bastos FI, Magnanini MMF, Silva CMFP. Iniciativa STROBE: subsídios para a comunicação de estudos observacionais. Rev Saúde Pública [Internet]. 2010 [cited 2021 Jul 29];44(3):559-65. Available from: https://www.strobestatement.org/fileadmin/Strobe/uploads/translations/STROBE_translation_portuguese_Commentary_Malta_RevSaudePublica_ 2010_checklist.pdf.

20. 20.Teich VD, Klajner S, Almeida FAS, Dantas ACB, Laselva CR, Torritesi MG et al. Características epidemiológicas e clínicas de pacientes com COVID-19 no Brasil. Einstein [Internet]. 2020 [cited 2021 Ago 03]; 18:eAO6022. DOI: https://doi.org/10.31744/einstein_journal/2020A06022.

21. Pauletti M, Otaviano MLPO, Moraes AST, Schneider DS. Perfil epidemiológico dos pacientes internados em um Centro de Terapia Intensiva. Aletheia [Internet]. 2017 [cited 2020 Oct 23]; 50(1-2):38-46 Available from: http://www.periodicos.ulbra.br/index.php/aletheia/article/view/4160/2987.

22. Albuquerque JM, Silva RFA, Souza RFF. Epidemiological profile and monitoring after discharge of patients hospitalized at an intensive care unit. Cogitare Enferm [Internet]. 2017 [cited 2020 Oct 25]; (22)3:e50609. DOI: http://dx.doi.org/10.5380/ce.v22i3.50609.

23. Instituto Brasileiro de Geografia e Estatística (IBGE). Projeções da população, Brasil e Unidades da Federação. Rio de Janeiro: IBGE 2013 (Série Relatórios Metodológicos, v. 40). 2013 [cited 2021 Jan 12]. Available from: https://www.ibge.gov.br/estatisticas/sociais/populacao/9109-projecao-da-populacao.html?edicao=9116.

24. Silva DF, Oliveira MLC. Epidemiology of COVID-19: comparison between epidemiological bulletins. Comun. Ciênc. Saúde [Internet]. 2020 [cited 2021 Jan 16]; 31(Suppl 1):61-74. Available from: http://www.escs.edu.br/revistaccs/index.php/comunicacaoemcienciasdasaude/article/view/661/293.

25. Tolentino ACMS, Peregrino AAF, Marta CB, Silva RCL, Schutz, $V$. The cost of the urinary catheterization in patients admitted to the intensive care unit. Rev. Fund. Care Online. [Internet]. 2017 [cited 2019 Jul 23]; 9(4):1170-6. DOI: https://doi.org/10.9789/2175-5361.2017.v9i4.1170-1176.

26. Mota EC, Oliveira AC. Catheter-associated urinary tract infection: why do not we control this adverse event? Rev. esc. enferm. USP [Internet]. 2019 [cited 2020 Nov 03]; 53:e03452. DOI: https://doi.org/10.1590/s1980-220x2018007503452.

27. Barbosa TP, Beccaria LM, Silva DC, Bastos AS. Association between sedation and adverse events in intensive care patients. Acta paul. Enferm. [Internet]. 2018 [cited 2020 Oct 01]; 31(2):194-200. DOI: https://doi.org/10.1590/1982-0194201800028.

28. Cavalcante TMC, Braquehais AR, Bezerra PARG. Bladder catheter delay: perfil epidemiological urinary infection in intensive therapy center. Rev. Tendên. Da Enferm. Profis. [Internet]. 2017 [cited 2020 Oct 15]; 9(2):2164-9. Available from: http://www.coren-ce.org.br/wp-content/uploads/2019/02/SONDA-VESICAL-DE-DEMORA-PERFIL-EPIDEMIOL\%C3\%93GICO-DAINFEC\%C3\%87\%C3\%830-URIN\%C3\%81RIA.pdf.

29. Borges DL, Rapello GVG, Deponti GN, Andrade FMD. Prone position in the treatment of acute respiratory failure due to COVID19. ASSOBRAFIR Ciência [Internet]. 2020. [cited 2020 Nov 03]; 11(Supl 1):111-20. DOI: http://dx.doi.org/10.47066/21779333.AC20.covid19.011. 
30. Constantin AG, Moreira APP, Oliveira JLC, Hofstätter LM, Fernandes LM. Incidência de lesão por pressão em unidade de terapia intensiva para adultos. ESTIMA, Braz. J. Enterostomal Ther. [Internet]. 2016 [cited 2020 Nov 29]; 16:e1118. DOI: https://doi.org/10.30886/estima.v16.454_PT.

31. Pachá HHP, Faria JIL, Oliveira KA, Beccaria LM. Pressure Ulcer in Intensive Care Units: a study control-case. Rev. Bras. Enferm. [Internet]. 2018 [cited 2020 Nov 01]; 71(6):3027-34. DOI: http://dx.doi.org/10.1590/0034-7167-2017-0950.

32. Barbosa TP, Beccaria LM, Poletti NAA. Pressure ulcer risk assessment in intensive care unit: preventive nursing care. Rev. Enferm. UERJ [Internet]. 2014 [cited 2021 Jan 10]; 22(3):353-8. Available from: https://www.epublicacoes.uerj.br/index.php/enfermagemuerj/article/view/13724.

33. Anghinoni TH, Contrin LL, Beccaria LM, Frutuoso IS, Rodrigues AMS, Wernek AL. Adherence to the protocol for the prevention of urinary tract infection. Rev. Enferm. UFPE [Internet]. 2018 [cited 2019 Sep 02]; 12(10):2675-82. DOI: https://doi.org/10.5205/1981-8963-v12i10a234874p2675-2682-2018.

34. Moraes A, Oliveira JLC, Fernandes LM, Tonini NS, Klassmann JC, Nicola AL. Assessment of the quality of nursing care in maintenance of long-term indwelling urinary catheters. Rev. Enferm. UFPE [Internet]. 2015 [cited 2019 Oct 22]; 9(10):9478-84. Available from: https://periodicos.ufpe.br/revistas/revistaenfermagem/article/viewFile/10890/12144.

35. Barros LAA, Paiva SS, Gonçalves Filho A, Sousa SMA. Risk nursing diagnostics for adverse events in bladder catheterization installation delay. Rev. enferm. UFPE [Internet]. 2016 [cited 2019 Oct, 26]; 10(9):3302-12. Available from: https://scholar.archive.org/work/kllnbhmaa5adho7qrvvqssjtye/access/wayback/https://periodicos.ufpe.br/revistas/revistaenf ermagem/article/download/11410/13188.

36. Soares TN, Pantoja CEF, Alves LLG, Alves LLG, Gomes AHM, Monteiro THA, et al. The influence of permanent education in the manipulation of delay vesical catheters. Rev. Eletronic. Acervo Saúde [Internet]. 2020 [cited 2020 Nov 01]; 12(10):e4476. DOI: https://doi.org/10.25248/reas.e4476.2020.

37. Gusmão GL, Silva LX, Azevedo AS. Assistência de Enfermagem no Tratamento da Trombose Venosa Profunda Em Pacientes Críticos. Persp. Online: biol. \& saúde [Internet]. 2014 [cited 2020 Oct 25]; 15(4):50-60. Available from: https://ojs3.perspectivasonline.com.br/biologicas_e_saude/article/view/533.

38. Melo FS, Azevedo SL, Porto IS, Tavares FG, Braga ALS, Paiva PB. Rational use of antimicrobials in the intensive care unit. Rev. Enferm. UFPE [Internet]. 2019 [cited 2020 Oct 10]; 13(5):1475-84. Available from: https://periodicos.ufpe.br/revistas/revistaenfermagem/article/view/238666/32331.

39. McCoy IE, Chertow GM, Chang TI-H. Patterns of diuretic use in the intensive care unit. PLoS ONE [Internet]. 2019 [cited 2020 Nov 01]; 14(5):e0217911 DOI: https://doi.org/10.1371/journal.pone.0217911. 